\title{
A modified technique for correction of trachomatous cicatricial entropion*
}

\author{
C. P. THOMMY \\ From the Guinness Ophthalmic Unit, Ahmadu Bello University Teaching Hospital, \\ Kaduna, Nigeria
}

SUMMARY Cicatricial entropion resulting from trachoma occurs in various grades of severity. No one technique of surgical correction is suitable for all types. The technique has to be modified in accordance with the severity of the condition. A modified technique using skin graft is described, and excellent results $(93.8 \%)$ in 380 lid corrections with minimal recurrences during a follow-up period of over 2 years are presented.

Entropion and trichiasis present in various grades of severity in areas where trachoma is endemic. No one method is suitable for surgical correction of these various grades of severity. This is why there are innumerable methods and modifications for entropion correction. A mild entropion may be corrected by a simple skin muscle excision, whereas a severely scarred lid calls for more radical methods with a combination of techniques. The choice of technique thus depends on the severity of entropion. Some of the available techniques are simple, others have more involved procedures, and some have certain drawbacks (Hadija, 1960) with consequent corneal changes. Some give higher success rates than others, depending on the aetiology and severity of entropion (Bercovici et al., 1977).

This paper presents a modified method for correcting extensive cicatricial entropion and trichiasis which is simple, has fewer drawbacks, and is followed by fewer recurrences.

\section{Material and methods}

380 eyes ( 360 bilateral, 20 unilateral) of 200 patients, predominantly female (170 females, 30 males) between the ages of 5 and 80 years (largest number between 15 and 56 years) were operated on for severe entropion/trichiasis resulting from trachomatous scarring. The operations were done at the Guinness Ophthalmic Unit of Ahmadu Bello Uni-

*This paper was presented in part at the 13th International Congress of Ophthalmology, Kyoto, Japan, 14-20 May 1978.

Correspondence to Dr Thommy. versity Teaching Hospital, Kaduna, Nigeria, from October 1976 to January 1979. 178 patients (341 eyes-326 bilaterally corrected and 15 unilaterally) were observed for a minimum period of 4 months and a maximum of 2 years and 4 months, $70 \%$ having been seen over $1 \frac{1}{2}$ years.

\section{Technique}

Under local infiltration anaesthesia for adults and general anaesthesia for children the lid is split intermarginally for its whole length with a No. 15 Bard Parker knife; a lid guard stretches the lid and protects the eyeball. The depth of the split depends on the mobilisation needed of the cilia-bearing flap, which in turn depends on the severity of entropion and trichiasis. The cilia roots, if any, on the posterior flap are excised completely. If the tarsal plate is very thickened and/or infected, it is sufficiently excised. At both ends, just beyond the last cilia, the incision is continued upwards for about 3 to $4 \mathrm{~mm}$, dividing the pretarsal orbicularis fibres and the anterior fibres of the muscle of Riolan. At the medial end care is taken to avoid the punctum (Fig. 1). Two parallel skin incisions are now made horizontally about 1.5 to $2 \mathrm{~mm}$ apart, the first being 3 to $4 \mathrm{~mm}$ above the lid margin and joining the 2 vertical cuts. This strip of skin is dissected free but not severed at either end, and brought down below the anterior flap, the attached ends passing through the vertical cuts (Fig. 2). A polyethylene tube is placed over this graft, and 4 to 5 mattress sutures with $4 / 0$ silk are taken to include the skin incisions, the graft, and the polyethylene tube. Care is taken to pass the sutures through the 


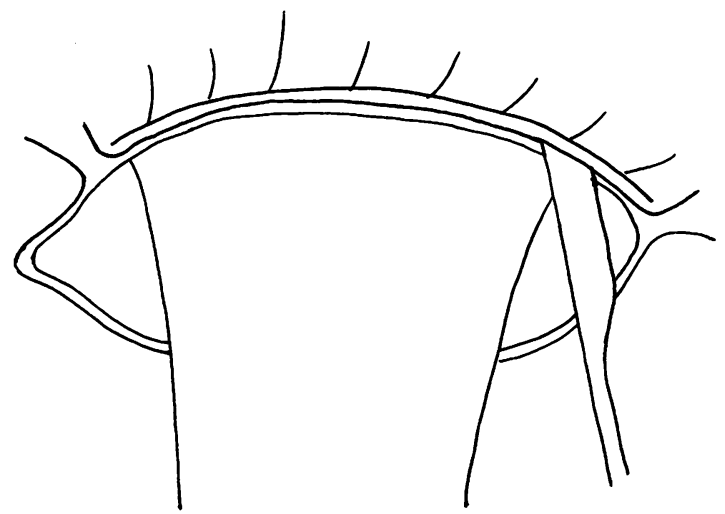

Fig. 1 The lid is split intermarginally and at each end continued upwards for 3-4 mm

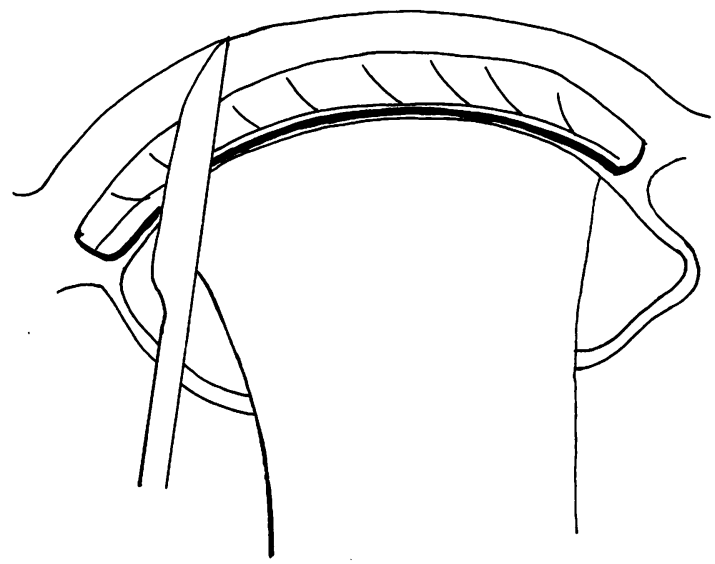

Fig. 2 The skin graft is dissected, the first incision joining the vertical cuts

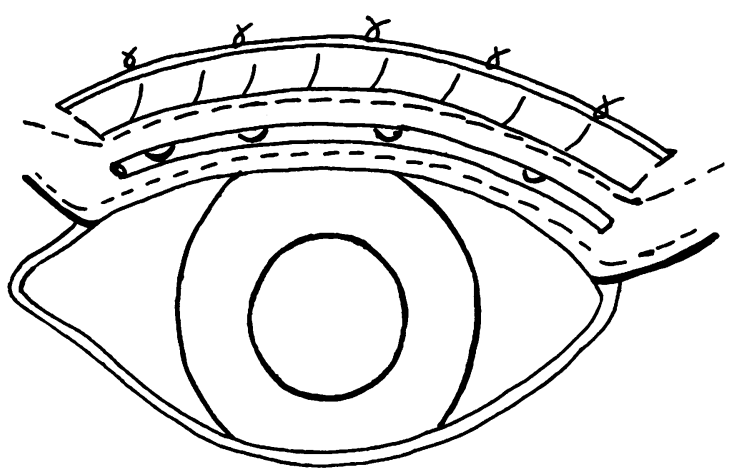

Fig. 3 The skin graft is sutured in the intermarginal space under a polyethylene tube, the unservered ends passing through the vertical cuts

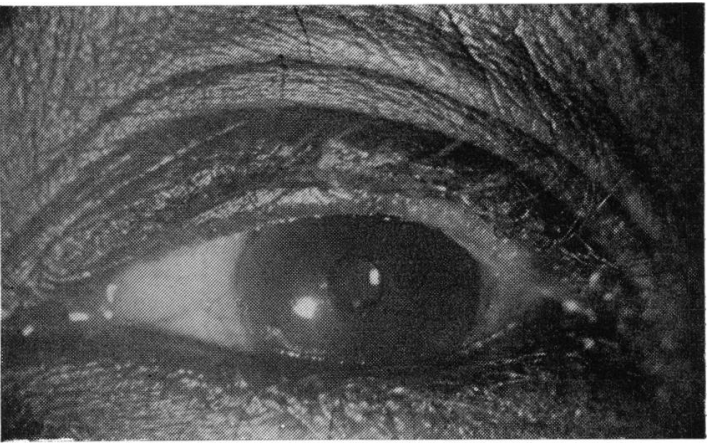

Fig. 4 The corrected eye

lower margin of the graft, and they are tied with optimum tension above the upper skin incision (Fig. 3).

Particular care is taken to excise completely all cilia roots from the posterior flap; to include the very last lash at both ends within the upward cuts; to have the graft lie properly in upward cuts at both ends; to pass sutures through the lower border of skin graft; and to leave about $2 \mathrm{~mm}$ of free edge of the posterior flap.

A pad and bandage is applied. Daily dressing is done for 3 days, after which dressing is discarded. Sutures and tube are removed on 7 th or 8th day.

\section{Results}

320 corrections out of 341 , followed up $(93.8 \%)$ were well maintained during the period of observation. There were no significant complications during or after surgery. There were no recurrences of entropion, but in a few cases $(21=6.6 \%)$ a few misdirected lashes were seen to be growing from the lower flap, which were promptly removed by electrolysis.

Subjective improvement of wellbeing, with absence of irritation, blepharospasm, photophobia, watering, discharge, and a gradual clearing of the cornea, were remarkable, as might be expected (Fig. 4).

\section{Discussion}

Extreme severity of trachomatous scarring calls for radical techniques and a combination of procedures for surgical correction (Callahan and Dortzbach, 1970), because these cases are often complicated by long-standing neglect, indigenous surgery and medication, and repeated attempts at correction. Some of these cases are amenable to tarsotomies (Hadija, 1960), including modifications of Weis's (Ballen, 1964; Sandford-Smith, 1976) or 
to tarsectomies (Boase, 1952; Jones et al., 1976). Grafting of split intermarginal strip with mucous membrane, with modifications (Spaeth, 1948) of Van Milligan's technique (Callahan and Dortzbach, 1970 ) or with skin by the modification by King and Wadsworth (1970) of Macheck-Blaskovic's technique are suitable in other cases.

The author further modifies the skin graft technique by making vertical cuts at both ends of the lids, also partially severing the pretarsal and Riolan's fibres. This procedure has the following advantages. Firstly, it facilitates better and easier mobilisation of the anterior cilia-bearing flap. Secondly, the descending attached portions of the skin graft at both ends can be perfectly placed in these cuts. This procedure prevents the formation of cysts and fistulae, gives a fine cosmetic result, bridges the gap between the cut ends of the pretarsal and anterior fibres of Riolan, removes the element of blepharospasm from the link of the vicious circle in the production and maintainance of entropion, helps healing during the immediate postoperative period, and maintains the correction later, preventing recurrence. It is also possible that it prevents the sliding of the preseptal orbicularis fibres over the pretarsal fibres (Jones and Wobing, 1976).

There was no recurrence of entropion in this series, so that the mechanical correction of entropion is fully successful. The $6.6 \%$ recurrence of trichiasis was because of nonexcision of all the cilia roots from the lower flap from where the misdirected lashes grew. This can therefore be prevented by complete excision of all the lash roots on the lower flap. Paring away of thickened and/or infected tarsal tissue leaves the desired thickness of the tarsus throughout its length for a nearly normal lid structure without any chance of vertical shortening of the lid.

The intention is always to make the intermarginal incision above the orifices of the Meibomian glands, but it is often difficult to carry out because of the altered anatomy of a scarred lid both from disease and previous treatment. However, no deleterious effect was seen on the cornea because of any possible alteration in the precorneal film.

The favourable results, absence of complications, and minimal recurrences of trichiasis may be attributed to the following factors: the vertical incisions severing the pretarsal and Riolan's fibres: better mobilisation of the anterior flap; the skin graft maintaining the correction; the use of a polyethylene tube for optimum positioning of the graft and the everted position of the cilia-bearing layer; the excision of the scarred tarsal plate and removal of all cilia roots from the lower flap; the mattress sutures along with the polyethylene tube enhancing the correction and maintaining it during the healing period; and the 'pedicled at both ends' skin graft, which always takes.

The method is simple, in that the graft is from the same lid. The polyethylene tube is readily available (it is smooth and pliable and cosmetically acceptable during the postoperative period). The procedure is short (average time 7 minutes), and it can be performed anywhere, usually as an outpatient procedure.

The only possible drawback to this procedure is the presence of minute downy hairs on the graft. This is prevented from rubbing the cornea by the anterior placement of the graft, taking the sutures at the lower border of the graft, suturing it to the anterior cilia-bearing layer, and leaving about $2 \mathrm{~mm}$ of free margin of the lower posterior flap.

The results are encouraging $(93.8 \%)$, and, if particular attention is paid to the details of the procedure, recurrences may be entirely avoided and the downy hairs prevented from rubbing on the cornea. Functionally it is satisfactory. Cosmetjcally, the method is excellent, the postoperative appearance being most natural. There is no staring, no etropion, and no vertical shortening of the lid.

\section{References}

Ballen, P. H. (1964). A simple procedure for the relief of trichiasis and entropion of the upper lid. Archives of Ophthalmology, 72, 239-240.

Bercovici, E., Hornblass, A., and Smith, B. (1977). Cicatricial entropion. Ophthalmic Surgery, 8, 112-115.

Boase, A. J. (1952). Tarsectomy. British Journal of Ophthalmology, 36, 645-648.

Callahan, A., and Dortzbach, R. K. (1970). Correction of cicatricial entropion of the upper lid. In Ophthalmic Plastic Surgery, Up to Date, 1st ed., Chapter 5, p. 76. Edited by J. C. Mustarde, L. T. Jones, and A. Callahan. Aesculapius Publishing Company: Birmingham, Alabama, USA.

Hadija, K. G. (1960). New method for the correction of entropion with trichiasis by tarsectomy. British Journal of Ophthalmology, 44, 436-439.

Jones, B. R., Barras, T. C., Hunter, P. A., Darougar, S., and Mohsenine, H. (1976). Neglected lid deformities causing progressive corneal disease. Surgical correction of entropion, trichiasis, marginal keratinization and functional lid shortening. Transactions of the Ophthalmological Societies of the UK, 96, 45-51.

Jones, L. T., and Wobing, J. L. (1976). In Surgery of the Eyelids and Lacrimal System, 1st ed., p. 74. Edited by L. T. Jones and J. L. Wobing. Aesculapius Publishing Company: Birmingham, Alabama, USA.

King, J. H., and Wadsworth, J. A. C. (1970). Surgery of eye lids. An Atlas of Ophthalmic Surgery, 2nd edn., Chapter 3, p. 46. Lippincot: Philadelphia.

Sandford-Smith, J. H. (1976). Surgical correction of trachomatous cicatrical entropion. British Journal of Ophthalmology, 60, 253-255.

Spaeth, E. B. (1948). Entropion and trichiasis. Principles and Practice of Ophthalmic Surgery, 4th edn., Chapter XII, p. 521. Henry Kimpton: London. 